\title{
Online parameters identification on first order delay temperature model for industrial brazing furnaces
}

\author{
Zheng De-xing, Wang Zhenggang \& Zhou Hai \\ School of UG, Yancheng Institute of Technology, Yancheng, P.R. China
}

KEYWORD: brazing furnace; temperature control; parameter identification; Recursive least squares algorithm;

ABSTRACT: In the brazing operation, the brazing temperature control is also the difficulty for its time delay and parameter varying. In order to control the brazing temperature, the mathematical model of brazing temperature must be established first. Parameter identification is an effective way to obtain the temperature model of brazing furnace. In this paper, the online parameter identification on first order delay temperature model for brazing furnaces was investigated. The recursive least squares algorithm with variable forgetting factor is applied to this study. Through identifying and calculating for first order delay temperature model, the parameters of the mode and delay time are got. This presents a solution on the time delay identification of traditional least squares algorithm, and solves the fuzzy identification resulted by data saturation.

\section{Introduction}

Brazing is a process to use weldment heat together with solder whose melting point lower than the base metal, after the solder melt, wetting and filling the gap that connecting the base metal with solder, solder metal and base metal to form a solid connection through diffusion (Zhuang M, Atherton D P. 1993; Merzagora A C, Bracchi F, Cerutti S, et al.2007; Zhang X P, Yu C B, Zhang Y P, et al. 2007; Zhang S Z, Kang G W.2011; ). The production of industrial brazing furnace process is a very complex physical process and chemical process. In the brazing operation, brazing temperature control is the key technology. Usually, according to the requirements of the process, the workpiece is needed to be heated gradually from the room temperature to $650^{\circ} \mathrm{C}$. In process, the temperature error is not more than $1{ }^{\circ} \mathrm{C}$, and the overshoot is not allowed. Then, the brazing temperature control is also the difficulty of brazing furnace for its time delay and parameter varying. So, several intelligent control methods such as chaos theory, adaptive artificial neural network, genetic algorithm, fuzzy theory, and so on have been used in the temperature control system of the large brazing furnaces(Hang $\mathrm{C} \mathrm{C}$, Astrom $\mathrm{K}$ J, Ho W K.1991; Park D J, Jun B E.1991; Zhuang M, Atherton D P.1993; Ang H K, Chong G, Li Y.2005; Paleologu C, Benesty J, Ciochina S.2008; Zhang X P, Yin L M, Yu CB. 2008; Zhao J, Cheng C, Qi L, et al. 2009).

In order to control the brazing temperature, the mathematical model of brazing temperature must be established first. System parameter identification is an effective way to obtain the temperature model. In this paper, the online parameters identification on first order delay temperature model for brazing furnaces was studied.

\section{Identification principle}

Identification is based on the input and output data to estimate the unknown parameters of the model under the guidance of the identification criteria. Parameter identification principle is as shown in Fig.1.

In Fig. $1, \theta$ is the parameter to be estimated, $\hat{\theta}$ is estimated value.

By the method of stepwise approximation, the current output value is calculated as follows:

$$
\hat{z}(k)=h^{T}(k) \hat{\theta}(k-1)
$$

The error value predicted is

$$
\widetilde{z}(k)=\mathrm{z}(k)-\hat{z}(k)
$$

Where $z(k)$ is the output in the process of parameters identification. 


$$
z(k)=h^{T}(k) \theta_{0}(k-1)+e(k)
$$

Where $h^{T}(k)$ is the direct measurement value.

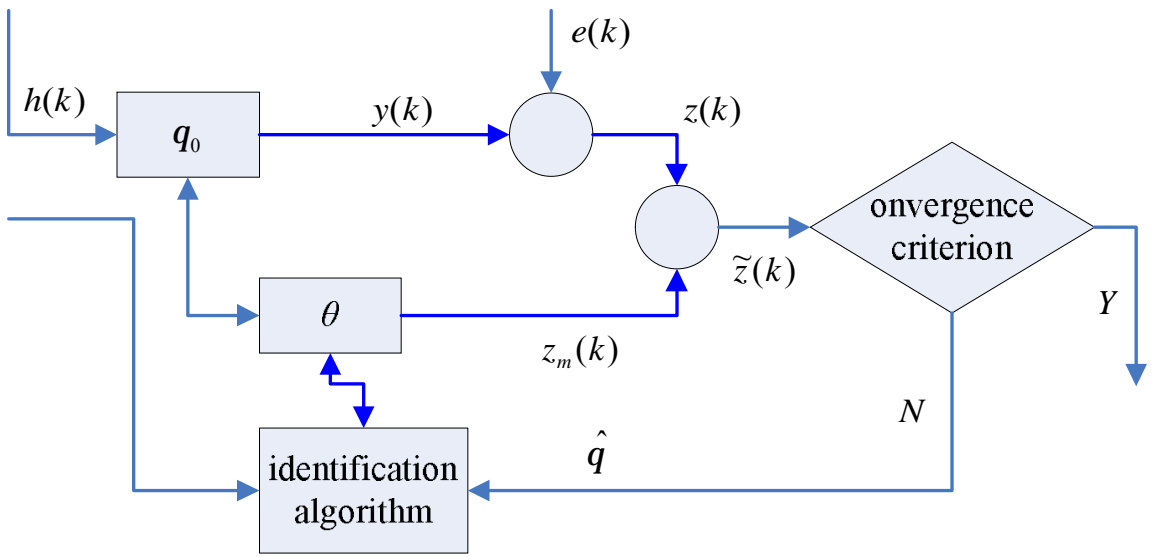

Fig.1 Identification principle

In the process of identification, $\hat{z}(k)$ gradually approaches $z(k)$. When this process is completed, the identification parameter model to substitute the actual model is got.

\section{Convergence criterion}

In the process of identification, the convergence criterion is applied to evaluate how the predictive value is approximated to the actual. Usually, the functional representation of the error is as follows:

$$
J(\theta)=\sum_{k=1}^{L} f(\varepsilon(k))
$$

Where $f(\varepsilon(k))=\varepsilon^{2}(k)$ is the squared error function, $\varepsilon(k)=z(k)-z_{m}(k)$ is the identification error. The output error model is presented in Fig.2.

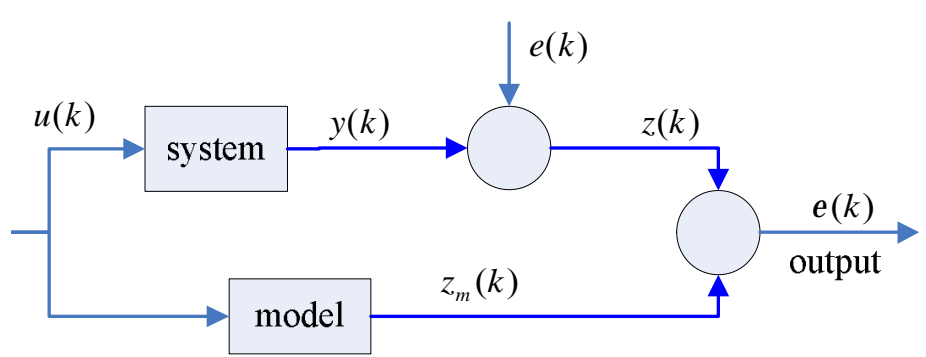

Fig.2 Output error map

\section{Parameter identification of first order delay temperature model}

\section{Discretization of temperature model}

In the brazing process, part of temperature step response shows a monotone convex trend. Then the mathematical model with the first order plus delay is adopted to express the response as:

$$
G(s)=K_{s} e^{-\tau s} / T_{s} s+1
$$

Here $K_{s}$ is the amplification factor, $T_{s}$ is the time constant and $\tau$ is the delay time.

Discrete $G(s)$, and add a sampling switch and a 0 - degree holder with the sampling period $T$ assumed, $G(s)$ is also written as,

$$
G_{h}(s)=1-\exp (-T \bullet s) / T_{s} s+1
$$

After discretization, $G(s)$ is 
$G\left(z^{-1}\right)=z^{-d}\left(1-e^{-T / T_{s}}\right) / z-e^{-T / T_{s}}$

After standardization, $G(s)$ is also written as,

$G\left(z^{-1}\right)=\frac{B\left(z^{-1}\right)}{A\left(z^{-1}\right)} \bullet z^{-d}=\frac{b z^{-1}}{1-a z^{-1}} \bullet z^{-d}$

Where $a=e^{-T / T_{s}}, b=K_{s}(1-a)$.

The above formula is converted to the differential equation as follows,

$y(k+1)=a y(k)+b u(k-d)$

Here, $a$ and $b$ are the process parameters to identify, $d$ is the time delay parameter to identify.

\section{Recursive least squares algorithm with variable forgetting factor}

$B\left(z^{-1}\right)$ of the temperature model are expanded to a polynomial, then $G(z)$ is converted to $G_{\mathrm{m}}(z)$.

$$
G_{m}(z)=\frac{B\left(z^{-1}\right)}{A\left(z^{-1}\right)} \bullet z^{-d}=\frac{b_{1} z^{-1}+b_{2} z^{-2}+\ldots \ldots+b_{m} z^{-m}}{1-a z^{-1}}
$$

According to the least squares algorithm, Formula 10 is written as,

$$
y(k)=h^{T}(k) \theta+e(k)
$$

In above formula, $y(k)$ is the observed data; $e(k)$ is the residual error whose average is 0 ; the estimated parameters are as follows:

$$
\begin{aligned}
& h(k)=\left[\begin{array}{l}
-y(k-1), u(k-1), u(k-2), \\
u(k-3), \ldots \ldots, u(k-m)_{m}
\end{array}\right]^{T} ; \\
& \theta=\left[a_{1}, b_{1}, b_{2}, \ldots \ldots, b_{m}\right]^{T} .
\end{aligned}
$$

Intergrate the forgetting factor, the convergence function of recursive least squares algorithm is

$$
J(\theta)=\sum_{k=1}^{L} \lambda(k)\left[y(k)-h^{T}(k) \theta\right]^{2}
$$

Then, $\left\{\begin{array}{c}\hat{\theta}(k+1)=\hat{\theta}(k)+K(k+1)\left[y(k+1)-h^{T}(k+1) \hat{\theta}(k)\right] \\ K(k+1)=P(k) h(k+1)\left[h^{T}(k+1) P(k) h(k+1)+\lambda(k)\right]^{-1} \\ P(k+1)=\frac{1}{\lambda(k)}\left[I-K(k+1) h^{T}(k+1)\right] P(k)\end{array}\right.$

Where $K(k)$ is the gain matrix, $P(k)$ is the covariance matrix.

The variable forgetting factor is as follows,

$$
\lambda(k)=\lambda_{\min }+\left(1-\lambda_{\min }\right)^{2^{L(n)}}
$$

Here $L(n)=\operatorname{INT}\left(\rho e(n)^{2}\right)$.

\section{Parameter identification of temperature model}

The principle on parameters identification is shown in Fig.3. $G(z)$ presents the actual model, $G_{\mathrm{m}}(z)$ is the identification object model. In process, the input data $u(k)$ is delivered to $G(z)$ and $G_{\mathrm{m}}(z)$, then $e(n)$ is got through $y(k)$ subtracting $y_{m}(k)$. Next, $\lambda(k)$ is considered in $e(n), J(\theta)$ is calculated. The identification procedures are as follows:

(1) Given initial conditions, and innitial the variable forgetting factor. 


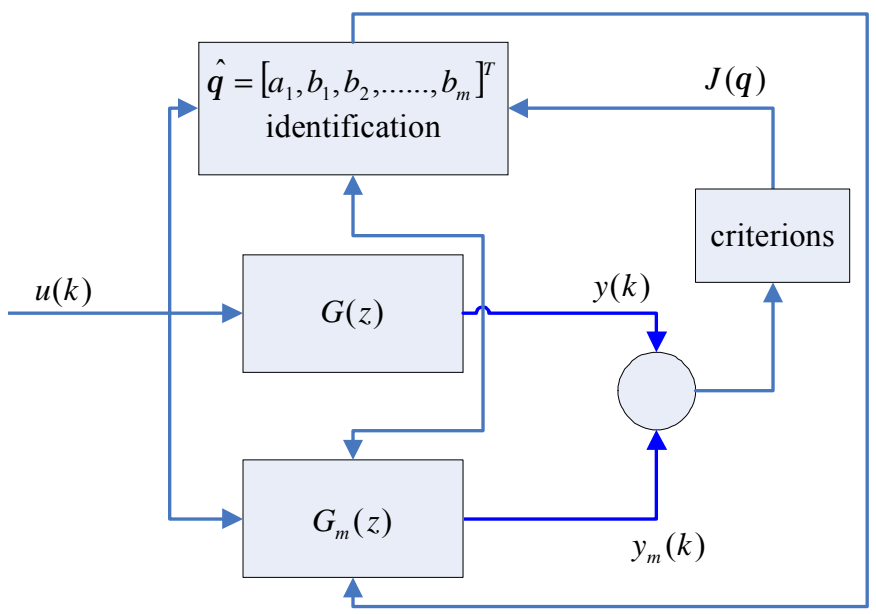

Fig.3 Algorithm structure diagram

(2)Based on $u(k)$ and $y(k)$, Construct the input vector $h(k)$.

(3) According to the least squares formula, calculate $P(k)$ and $K(k)$.

(4) Estimate $a_{1}, b_{1}, b_{2}, \ldots \ldots, b_{m}$.

(5) Calculate $b$ and $d$.

(6) Repeat from Step 2 to Step 5, assignment to $k=k+1$.

(7) Finish heating and end identifying.

In order to verify the identification algorithm, a simulation experiment was implemented in Matlab. The actual temperature curve is compared to the identification curve in Fig.4 and Fig.5 shown.

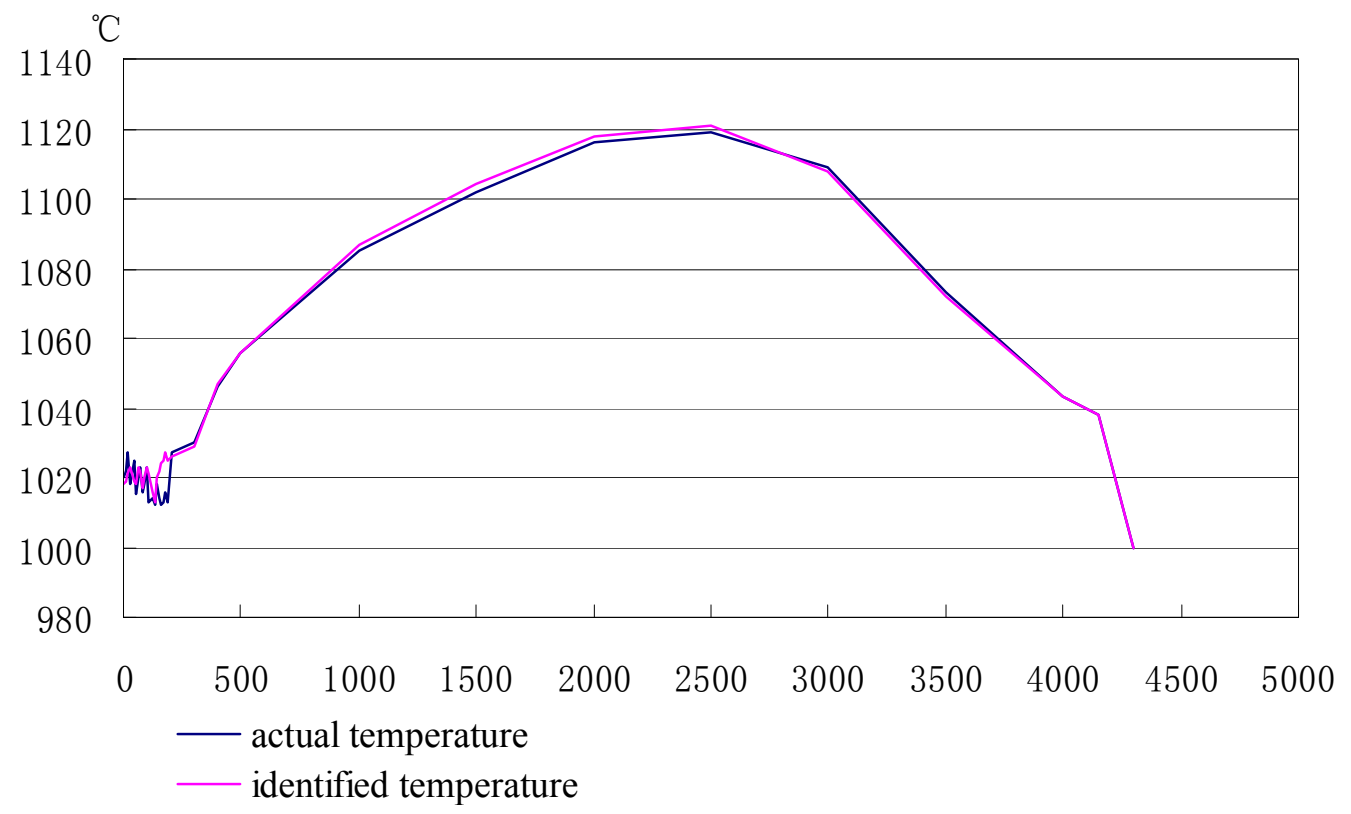

Fig.4 Comparation between actual temperature and identified temperature 


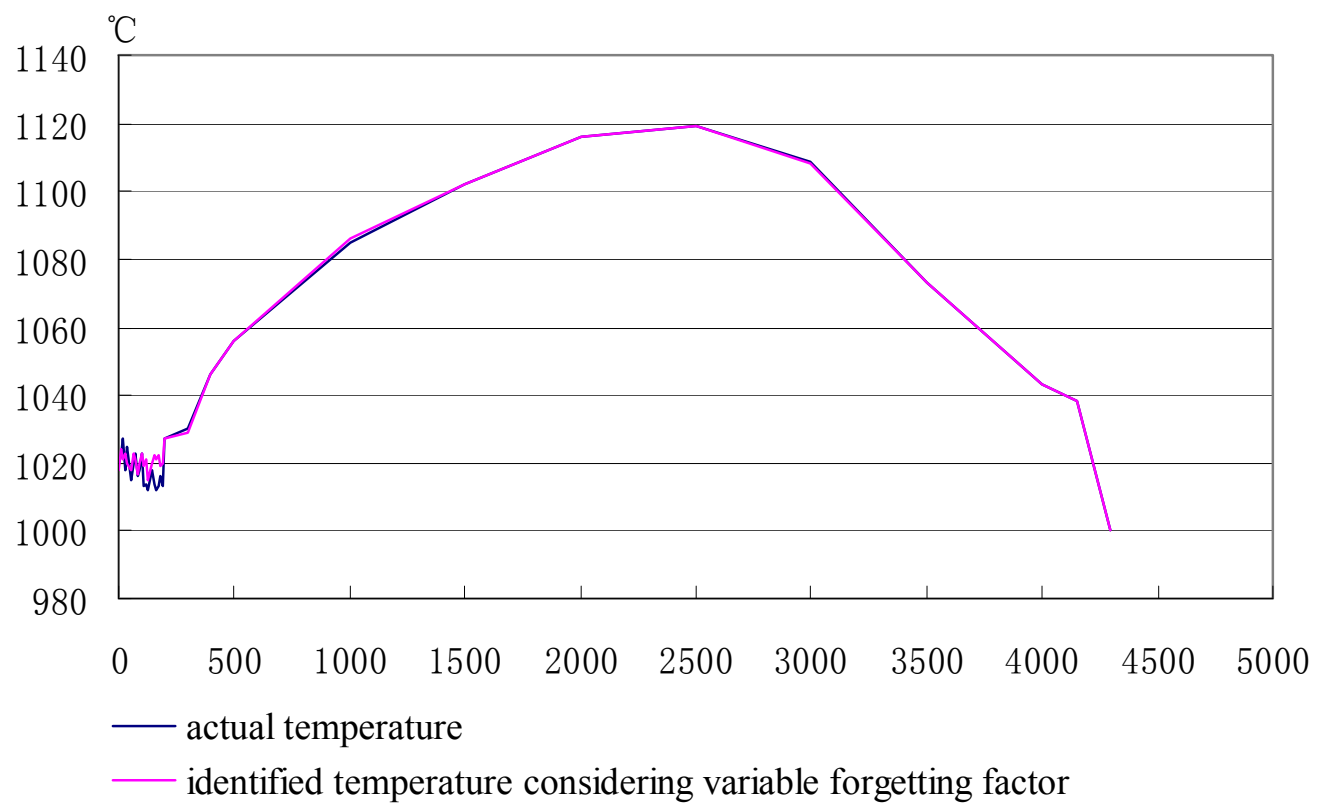

Fig.5 Comparation between actual temperature and identified temperature considering variable forgetting factor

Compare Fig. 4 to Fig.5, the result is shown that the temperature fluctuation is large in about 200, then tends to be gentle. From nearly 2500 , the actual temperature curve coincides with the identified temperature curves.

\section{Conclusions}

Parameter identification is an effective way to obtain the temperature model brazing furnace. This paper presents an online parameter identification study on first order delay temperature model for brazing furnaces. Through the parameter identification and calculation for first order delay temperature model, the parameters of the mode and delay time are got. This presents a solution on the time delay identification of traditional least squares algorithm, and solves the fuzzy identification resulted by data saturation. The simulation experiments verify the good identification accuracy and speed.

\section{Acknowledgements}

This work was funded by Jiangsu Province Research Joint Innovation Fund of China (No. BY2014108-24). The supports are gratefully acknowledged. The authors would like to thank all anonymous reviewers for their constructive suggestions.

\section{References}

[1] Ang H K, Chong G, Li Y. 2005. PID Control Systems Technology, IEEE Transactions System Analysis, Design, and Technology. Control on,13(4):559-576.

[2] Hang C C, Astrom K J, Ho W K. 1991. Refinement of the Ziegler-Nichols tuning formula. Control Theory and Applications, IEE Proceeding D, 138(2):111-118.

[3] Merzagora A C, Bracchi F, Cerutti S, et al. 2007. Evaluation and application of a RBF neural network for online single-sweep extraction of SEPs during scoliosis surgey. Biomedical Engineering, IEEE Transactions on, 54(7): 1300-1308.

[4] Paleologu C, Benesty J, Ciochina S. 2008. A robust variable forgetting factor recursive least-squares algorithm for system identification. Signal Processing Letters, IEEE, 15:597-600.

[5] Park D J, Jun B E. 1991. Fast tracking RLS algorithm using novel variable forgetting factor with unity zone. Electronics Letters, 27(23):1940-1943.

[6] Zhuang M, Atherton D P. 1993. Automatic turning of optimum PID controllers. Control Theory and Applications, IEE Proceeding D, 140(3):216-224. 
[7] Zhang X P, Yu C B, Zhang Y P, et al. 2007. Processing treatment of a lead-free Sn-Ag-Cu-Bi solder by rapid laser-beam reflowing and the creep property of its soldered connection. Journal of Materials Processing Technology, 192/193: 539-542.

[8] Zhang X P, Yin L M, Yu CB. 2008. Thermal creep and fracture behaviors of the lead-free $\mathrm{Sn}-\mathrm{Ag}$-Cu-Bi solder interconnections under different stress levels. Journal of Materials Science: Materials in Electronics, 19(4): 393-398.

[9] Zhang S Z, Kang G W. 2011. Research on intelligent control strategy of temperature system of industrial brazing furnac. Proceedings-IEEE 2011 10th International Conference on Electronic Measurement and Instruments, ICEMI 2011, v2, p 65-67.

[10]Zhao J, Cheng C, Qi L, et al. 2009. Kinetics of intermetallic compound layers and shear strength in Bi-bearing $\mathrm{SnAgCu} / \mathrm{Cu}$ soldering couples. Journal of Alloys and Compounds, 473 (1/2): 382-388.

[11] 